\section{Sum-Capacity Computation for the Gaussian Vector Broadcast Channel Via Dual Decomposition}

\author{
Wei Yu, Member, IEEE
}

\begin{abstract}
A numerical algorithm for the computation of sum capacity for the Gaussian vector broadcast channel is proposed. The sum capacity computation relies on a duality between the Gaussian vector broadcast channel and the sum-power constrained Gaussian multiple-access channel. The numerical algorithm is based on a Lagrangian dual decomposition technique and it uses a modified iterative water-filling approach for the Gaussian multiple-access channel. The algorithm converges to the sum capacity globally and efficiently.
\end{abstract}

Index Terms-Broadcast channel, duality, multiple-access channel, multiple-antenna.

\section{INTRODUCTION}

Consider a Gaussian vector broadcast channel:

$$
\boldsymbol{Y}=H^{T} \boldsymbol{X}+\boldsymbol{Z}
$$

where the vector $X$ represents the transmit signal, the matrix $H^{T}=$ $\left[H_{1} \cdots H_{K}\right]^{T}$ represents the channels for users $1 \cdots K$, the vector $\boldsymbol{Y}=\left[\boldsymbol{Y}_{1} \cdots \boldsymbol{Y}_{K}\right]^{T}$ represents receive signals for users $1 \cdots K$, and $\boldsymbol{Z}=\left[\boldsymbol{Z}_{1} \cdots \boldsymbol{Z}_{K}\right]^{T}$ is the additive independent and identically distributed (i.i.d.) Gaussian vector noise with unit variance on each of its components. Independent information is transmitted to each user. The Gaussian vector broadcast channel is not degraded; its capacity region has been established recently [1], [2], [4], [3], [5].

One of the techniques for establishing the broadcast channel capacity is an uplink-downlink duality relation between the Gaussian vector broadcast channel and a dual Gaussian vector multiple-access channel [3]-[5]. The duality result can be stated as follows. The capacity region of a Gaussian vector broadcast channel (1) is exactly the same as that of a dual multiple-access channel with $\tilde{X}=\boldsymbol{Y}$ as the transmitters, $H$ as the channel matrix, $\tilde{\boldsymbol{Y}}=\boldsymbol{X}$ as the receiver, and $\tilde{Z}$ as the unit-variance additive white Gaussian vector noise

$$
\tilde{\boldsymbol{Y}}=H \tilde{\boldsymbol{X}}+\tilde{\boldsymbol{Z}}
$$

In addition, the dual vector multiple-access channel has a sum power constraint across all transmitters rather than a set of individual power constraints on each transmitter. Uplink-downlink duality is useful because the input optimization problem for the Gaussian multiple-access channel is numerically easier to solve.

More explicitly, the Gaussian multiple-access channel with vector inputs and a vector output can be represented as follows:

$$
\tilde{\boldsymbol{Y}}=\sum_{k=1}^{K} H_{k} \tilde{\boldsymbol{X}}_{k}+\tilde{\boldsymbol{Z}}
$$

Manuscript received June 2, 2004; revised August 23, 2005. This work was supported in part by the Natural Science and Engineering Research Council (NSERC) of Canada and in part by the Canada Research Chairs program. The material in this correspondence was presented at the 37th Annual Conference on Information Science and Systems (CISS), Baltimore, MD, March 2003.

The author is with The Edward S. Rogers, Sr. Department of Electrical and Computer Engineering, University of Toronto, Toronto, ON M5S 3G4, Canada (e-mail: weiyu@ @omm.utoronto.ca).

Communicated by M. Médard, Associate Editor for Communications.

Digital Object Identifier 10.1109/TIT.2005.862106 where $\tilde{\boldsymbol{X}}_{k}$ 's are $n \times 1$ vector inputs, $H_{k}$ are $m \times n$ channel matrices, $\tilde{\boldsymbol{Y}}$ is the $m \times 1$ vector output, and $\tilde{\boldsymbol{Z}}$ is a $m \times 1$ additive Gaussian noise vector. The inputs $\tilde{\boldsymbol{X}}_{k}$ must be independent. In a usual multiple-access channel, a separate input power constraint is applied to each of $\tilde{\boldsymbol{X}}_{k}$, i.e.,

$$
\mathbb{E}\left[\tilde{X}_{\boldsymbol{k}}^{T} \tilde{\boldsymbol{X}}_{k}\right] \leq \mathrm{P}_{\mathrm{k}}, \quad k=1, \ldots, K .
$$

To utilize uplink-downlink duality, a different power constraint is applied. While retaining the assumption that $\tilde{\boldsymbol{X}}_{k}$ are independent, a sumpower constraint is applied to all $\tilde{\boldsymbol{X}}_{k}$ 's at the same time

$$
\sum_{k=1}^{K} \mathbb{E}\left[\tilde{\boldsymbol{X}}_{\boldsymbol{k}}^{T} \tilde{\boldsymbol{X}}_{k}\right] \leq \mathrm{P} .
$$

This correspondence proposes efficient numerical algorithms that compute the sum capacity of a Gaussian vector broadcast channel by computing the sum capacity of its dual Gaussian vector multiple-access channel with a sum-power constraint.

The capacity region of a multiple-access channel under a fixed input distribution $p\left(\tilde{\boldsymbol{x}}_{1}\right) \cdots p\left(\tilde{\boldsymbol{x}}_{K}\right)$ is a well-known pentagon region. In particular, the sum capacity of the multiple-access channel is the solution to the following mutual information maximization problem:

$$
C=\max _{\operatorname{co}\left\{p\left(\tilde{\boldsymbol{x}}_{\mathbf{1}}\right) \cdots p\left(\tilde{\boldsymbol{x}}_{\boldsymbol{K}}\right)\right\}} I\left(\tilde{\boldsymbol{X}}_{\mathbf{1}} \cdots \tilde{\boldsymbol{X}}_{\boldsymbol{K}} ; \tilde{\boldsymbol{Y}}\right)
$$

where $\operatorname{co}\{\cdot\}$ denotes the convex hull operation and the maximization is over the convex hull of all input distributions that satisfy the input constraint. For the Gaussian vector multiple-access channel, Gaussian inputs are optimal and the mutual information maximization problem can be cast as a convex optimization problem. More precisely, let $S_{k}=$ $\mathbb{E}\left[\tilde{\boldsymbol{X}}_{\boldsymbol{k}} \tilde{\boldsymbol{X}}_{\boldsymbol{k}}^{\boldsymbol{T}}\right]$ be the input covariance matrix for user $k$. The mutual information maximization problem under separate power constraints (4) becomes

$$
\begin{array}{ll}
\operatorname{maximize} & \frac{1}{2} \log \frac{\left|\sum_{k=1}^{K} H_{k} S_{k} H_{k}^{T}+S_{z}\right|}{\left|S_{z}\right|} \\
\text { subject to } & \operatorname{tr}\left(S_{k}\right) \leq \mathrm{P}_{\mathrm{k}}, \quad k=1, \ldots, K, \\
& S_{k} \geq 0, \quad k=1, \ldots, K .
\end{array}
$$

The maximization problem under the sum power constraint (5) becomes

$$
\begin{array}{ll}
\text { maximize } & \frac{1}{2} \log \frac{\left|\sum_{k=1}^{K} H_{k} S_{k} H_{k}^{T}+S_{z}\right|}{\left|S_{z}\right|} \\
\text { subject to } & \sum_{k=1}^{K} \operatorname{tr}\left(S_{k}\right) \leq \mathrm{P} \\
& S_{k} \geq 0, \quad k=1, \ldots, K
\end{array}
$$

where $\operatorname{tr}(\cdot)$ denotes the matrix trace operation and $S_{z}$ is the covariance matrix of $\tilde{Z}$. Because $\log |\cdot|$ is a concave function over the set of positive semidefinite matrices and constraints are linear, both problems belong to the class of convex optimization problems for which numerical solutions are in principle easy to obtain.

In particular, with a separate power constraint on each $\tilde{\boldsymbol{X}}_{k}$, the sum capacity problem (7) can be solved efficiently using an algorithm called "iterative water-filling" [6]. The key observation is that the maximization problem can be solved by iteratively updating one $S_{k}$ at a time while keeping all other $S_{k}$ 's fixed. Because the constraints are separable, each update can be done independently, and the iterative process converges to the sum capacity. However, this iterative procedure is not directly applicable to the sum-power problem (5) in which a coupled 
power constraint applies to all $\tilde{\boldsymbol{X}}_{k}$ 's at the same time. The main idea of this correspondence is that via a dual decomposition technique, the sum-power constraint may be decoupled. This allows us to take advantage of the efficiency of iterative water-filling.

The numerical solution for the sum-power constrained multiple-access channel has been considered in [7] where two algorithms also based on iterative water-filling are proposed for the sum-power problem. The algorithm proposed in this correspondence differs from that of [7] in that it utilizes the dual decomposition technique popularized by recent advances in network optimization (e.g., [8]). As the numerical results contained in this correspondence show, the dual decomposition approach has a lower complexity, a superior convergence rate and is more scalable with the problem size as compared with the algorithms proposed in [7].

The capacity computation for the Gaussian vector broadcast channel has also been considered in [9] in which a gradient ascent algorithm is proposed. Further, this problem can be solved using generic convex optimization methods such as the interior-point method as well. The iterative water-filling-based algorithms proposed in this correspondence and in [7] take advantage of the specific structure of the Gaussian mutual information maximization problem and have a faster convergence in practice. However, the iterative water-filling-based algorithms are also more specific as they work only for sum capacity and not for the entire capacity region.

The rest of the correspondence is organized as follows. The numerical solution for the sum-capacity problem based on the dual decomposition method and its convergence proof are presented in Section II. Simulation results and comparison with existing algorithms are presented in Section III. Conclusions are drawn in Section IV.

\section{AlgORIthm}

\section{A. Dual Decomposition}

Consider the optimization problem (8)

$$
\begin{array}{ll}
\operatorname{maximize} & \frac{1}{2} \log \frac{\left|\sum_{k=1}^{K} H_{k} S_{k} H_{k}^{T}+S_{z}\right|}{\left|S_{z}\right|} \\
\text { subject to } & \sum_{k=1}^{K} \operatorname{tr}\left(S_{k}\right) \leq \mathrm{P} \\
& S_{k} \geq 0, \quad k=1, \ldots, K
\end{array}
$$

where the optimization variables are semidefinite matrices $S_{1}, \ldots, S_{K}$. The optimization variable can also be thought of as $S=\operatorname{diag}\left\{S_{1}, \ldots, S_{K}\right\}$ with the constraint $\operatorname{tr}(S) \leq \mathrm{P}$. Without the constraint that $S$ must be block-diagonal, the problem is equivalent to a conventional Gaussian vector channel for which the well-known water-filling solution applies. However, water-filling does not necessarily result in a diagonal transmit covariance matrix. On the other hand, if $S$ is kept as diagonal, but individual power constraints are applied to each of $S_{k}$ rather than the sum power constraint, a numerical algorithm called "iterative water-filling" can be used to find the sum capacity efficiently [6]. As mentioned earlier, the idea of iterative water-filling is to optimize each $S_{k}$ individually while keeping all other $S_{k}$ 's fixed. The fixed point of the iterative algorithm is the global optimum. The primary difficulty in solving (9) is that while the transmit signals must be independent, the constraint on their covariance matrices is coupled.

The main idea of this correspondence is a dual decomposition technique that decouples the coupled constraint via a dual variable. The dual decomposition method works as follows. First, introduce a new set of scalar variables $\left\{p_{1} \cdots p_{K}\right\}$, and rewrite the optimization problem (9) in the following form which has only a single coupled constraint

$$
\begin{array}{ll}
\text { maximize } & \frac{1}{2} \log \frac{\left|\sum_{k=1}^{K} H_{k} S_{k} H_{k}^{T}+S_{z}\right|}{\left|S_{z}\right|} \\
\text { subject to } & \operatorname{tr}\left(S_{k}\right) \leq p_{k} \quad k=1, \ldots, K, \\
& S_{k} \geq 0, \quad k=1, \ldots, K, \\
& \sum_{k=1}^{K} p_{k} \leq \mathrm{P} .
\end{array}
$$

Form the Lagrangian of the optimization problem with respect to the coupled constraint $\sum_{k=1}^{K} p_{k} \leq \mathrm{P}$

$$
\begin{aligned}
& L\left(S_{1}, \ldots, S_{K}, p_{1}, \ldots, p_{K}, \lambda\right)= \\
& \quad \frac{1}{2} \log \frac{\left|\sum_{k=1}^{K} H_{k} S_{k} H_{k}^{T}+S_{z}\right|}{\left|S_{z}\right|}-\lambda\left(\sum_{k=1}^{K} p_{k}-\mathrm{P}\right) .
\end{aligned}
$$

Let the dual objective be

$$
g(\lambda)=\max _{S_{1}, \ldots, S_{K}, p_{1}, \ldots, p_{K}} L\left(S_{1}, \ldots, S_{K}, p_{1}, \ldots, p_{K}, \lambda\right)
$$

where the constraints of the maximization $\operatorname{are} \operatorname{tr}\left(S_{k}\right) \leq p_{k}$ and $S_{k} \geq 0$. Because the original optimization problem is convex, the dual objective reaches a minimum at the optimal value of the primal problem. Thus, the sum-power multiple-access channel sum capacity problem is equivalent to:

$$
\begin{array}{ll}
\operatorname{minimize} & g(\lambda) \\
\text { subject to } & \lambda \geq 0 .
\end{array}
$$

The key observations are that $g(\lambda)$ is easy to compute and the above dual minimization problem can be solved more efficiently than the original problem.

Consider first the evaluation of $g(\lambda)$. By definition, $g(\lambda)$ is the solution to the following optimization problem:

$$
\begin{array}{ll}
\text { maximize } & \frac{1}{2} \log \frac{\left|\sum_{k=1}^{K} H_{k} S_{k} H_{k}^{T}+S_{z}\right|}{\left|S_{z}\right|}-\lambda\left(\sum_{k=1}^{K} p_{k}-\mathrm{P}\right) \\
\text { subject to } & \operatorname{tr}\left(S_{k}\right)-p_{k} \leq 0, \quad k=1, \ldots, K \\
& S_{k} \geq 0, \quad k=1, \ldots, K
\end{array}
$$

Notice that the above maximization problem has decoupled constraints. Therefore, an iterative-water-filling-like algorithm can be used to solve the problem efficiently. The iterative algorithm works as follows. In each step, the objective function is maximized over a single pair of $\left(S_{k}, p_{k}\right)$, while keeping all other $\left(S_{k}, p_{k}\right)$ 's fixed. Since the objective is nondecreasing with each iteration, the algorithm must converge to a fixed point. At the fixed point, the set of $\left(S_{k}, p_{k}\right)$ satisfies the Karush-Kuhn-Tucker (KKT) condition of the optimization problem (14). Thus, the fixed point is the optimal solution.

In fact, each step of the iterative algorithm is just an evaluation of the water-filling covariance matrix with a fixed water level. Without loss of generality, consider the optimization over $\left(S_{1}, p_{1}\right)$ while keeping all other $\left(S_{k}, p_{k}\right)$ 's fixed. The KKT condition for the optimization problem (14) over $\left(S_{1}, p_{1}\right)$ (while keeping all other $\left(S_{k}, p_{k}\right)$ 's fixed) can be obtained by differentiating its Lagrangian with respect to $S_{1}$ and $p_{1}$. The Lagrangian is

$$
\begin{aligned}
\tilde{L}\left(S_{1}, p_{1}, \nu_{1}, \Phi_{1}\right)=\frac{1}{2} \log \frac{\left|\sum_{k=1}^{K} H_{k} S_{k} H_{k}^{T}+S_{z}\right|}{\left|S_{z}\right|} \\
-\lambda\left(\sum_{k=1}^{K} p_{k}-\mathrm{P}\right)-\nu_{1}\left(\operatorname{tr}\left(S_{1}\right)-p_{1}\right)-\operatorname{tr}\left(\Phi_{1} S_{1}\right) .
\end{aligned}
$$

where $\nu_{1}$ is the dual variable associated with the constraint $\operatorname{tr}\left(S_{1}\right)-$ $p_{1} \leq 0$, and $\Phi_{1}$ is the dual variable associated with the constraint 
$S_{1} \geq 0$. The KKT condition is obtained by setting $\partial \tilde{L} / \partial S_{1}=0$ and $\partial \tilde{L} / \partial p_{1}=0$. This gives

$$
\frac{1}{2} H_{1}^{T}\left(\sum_{k=1}^{K} H_{k} S_{k} H_{k}^{T}+S_{z}\right)^{-1} H_{1}=\nu_{1} I+\Phi_{1}
$$

and

$$
\nu_{1}=\lambda \text {. }
$$

The above two equations are equivalent to

$$
\frac{1}{2} H_{1}^{T}\left(\sum_{k=1}^{K} H_{k} S_{k} H_{k}^{T}+S_{z}\right)^{-1} H_{1}=\lambda I+\Phi_{1}
$$

which is exactly the water-filling condition for a Gaussian vector channel, except that in this case, $\lambda$ is fixed. Thus, the usual water-filling procedure only needs to be modified slightly in order to find the optimal $\left(S_{1}, p_{1}\right)$. The procedure is to water-fill up to a fixed water level, rather than to water-fill subject to a fixed total power. More precisely, let

$$
\left(\sum_{k=2}^{K} H_{k} S_{k} H_{k}^{T}+S_{z}\right)=Q^{T} \Lambda Q
$$

be an eigenvalue decomposition, where $\Lambda$ is a diagonal matrix and $Q$ is an orthogonal matrix. The maximization problem (14) over $\left(S_{1}, p_{1}\right)$ while keeping all other $\left(S_{k}, p_{k}\right)$ 's fixed is equivalent to the maximization of

$$
\frac{1}{2} \log \left|\Lambda^{-\frac{1}{2}} Q H_{1} S_{1} H_{1}^{T} Q^{T} \Lambda^{-\frac{1}{2}}+I\right|-\lambda p_{1}
$$

Let

$$
\Lambda^{-\frac{1}{2}} Q H_{1}=U\left[\begin{array}{ccc}
s_{1,1} & & \\
& \ddots & \\
& & s_{1, n}
\end{array}\right] V^{T}
$$

be a singular-value decomposition, where $U$ and $V$ are orthogonal matrices. The optimal $S_{1}$ is then

$$
S_{1}=V\left[\begin{array}{lll}
\left(\frac{1}{2 \lambda}-\frac{1}{s_{1,1}^{2}}\right)_{+} & & \\
& \ddots & \\
& & \left(\frac{1}{2 \lambda}-\frac{1}{s_{1, n}^{2}}\right)_{+}
\end{array}\right] V^{T} .
$$

The optimal $p_{1}$ is then

$$
p_{1}=\sum_{j=1}^{n}\left(\frac{1}{2 \lambda}-\frac{1}{s_{1, j}^{2}}\right)_{+}
$$

where $(\cdot)_{+}=\min (0, \cdot)$.

The next step is to use the same procedure to find the optimal $\left(S_{2}, p_{2}\right)$, while keeping $\left(S_{1}, p_{1}\right),\left(S_{3}, p_{3}\right), \ldots,\left(S_{K}, p_{K}\right)$ fixed. The algorithm then successively updates $\left(S_{3}, p_{3}\right),\left(S_{4}, p_{4}\right), \ldots$, then $\left(S_{1}, p_{1}\right),\left(S_{2}, p_{2}\right) \cdots$. The iterative procedure is guaranteed to converge to the optimal solution of (14).

It remains to minimize $g(\lambda)$ subject to the constraint $\lambda \geq 0$. Here, $g(\lambda)$ is a convex function. Further, the constraint set is a one-dimensional interval. Thus, a standard search algorithm on $\lambda$ yields satisfactory results. However, $g(\lambda)$ is not necessarily differentiable, so it is not always possible to take its gradient. Nevertheless, the structure of $g(\lambda)$ (i.e., the solution of (14)) reveals a possible search direction. In particular, it is possible to find a subgradient $h$ such that for all $\lambda^{\prime} \geq 0$

$$
g\left(\lambda^{\prime}\right) \geq g(\lambda)+h \cdot\left(\lambda^{\prime}-\lambda\right) .
$$

Let $\left(S_{1}^{*}, \ldots, S_{K}^{*}, p_{1}^{*}, \ldots, p_{K}^{*}\right)$ be the optimizing solution of $(14)$ in the definition of $g(\lambda)$. It is not difficult to see that

$$
\begin{aligned}
g\left(\lambda^{\prime}\right) & =\max \left\{\frac{1}{2} \log \frac{\left|\sum_{k=1}^{K} H_{k} S_{k} H_{k}^{T}+S_{z}\right|}{\left|S_{z}\right|}-\lambda^{\prime}\left(\sum_{k=1}^{K} p_{k}-\mathrm{P}\right)\right\} \\
& \geq \frac{1}{2} \log \frac{\left|\sum_{k=1}^{K} H_{k} S_{k}^{*} H_{k}^{T}+S_{z}\right|}{\left|S_{z}\right|}-\lambda^{\prime}\left(\sum_{k=1}^{K} p_{k}^{*}-\mathrm{P}\right) \\
& =g(\lambda)+\left(\mathrm{P}-\sum_{k=1}^{K} p_{k}^{*}\right)\left(\lambda^{\prime}-\lambda\right) .
\end{aligned}
$$

Thus, the following choice of $h$

$$
h=\mathrm{P}-\sum_{k=1}^{K} p_{k}^{*}
$$

is a subgradient of $g(\lambda)$. The subgradient search suggests that

$$
\begin{aligned}
& \text { increase } \lambda \text { if } \sum_{k=1}^{K} p_{k}^{*}>\mathrm{P} \\
& \text { decrease } \lambda \text { if } \sum_{k=1}^{K} p_{k}^{*}<\mathrm{P} .
\end{aligned}
$$

The search direction is intuitively obvious as $\lambda$ is related to the waterfilling level. The water-filling level should be adjusted according to whether the total power constraint is exceeded. Because $\lambda$ adjustment occurs in a one-dimensional space, it can be done efficiently using a bisection search.

An outline of the proposed algorithm is as follows:

Algorithm 1: Computation of the sum-power constrained Gaussian multiple-access channel sum capacity:

1) Initialize $\lambda_{\min }$ and $\lambda_{\max }$.

2) Set $\lambda=\left(\lambda_{\min }+\lambda_{\max }\right) / 2$.

3) Solve for $\left(S_{k}^{*}, p_{k}^{*}\right)_{k=1}^{K}$ in the optimization problem (14) with the above $\lambda$ by iteratively optimizing each of $\left(S_{k}, p_{k}\right)$ using (19)-(23) while keeping all other $\left(S_{k}, p_{k}\right)$ 's fixed. The iterations cycle through $k=1, \ldots, K, 1, \ldots, K, \ldots$ until the optimal solution of (14) is reached.

4) If $\sum_{k=1}^{K} p_{k}^{*}>\mathrm{P}$, then set $\lambda_{\min }=\lambda$, else set $\lambda_{\max }=\lambda$.

5) If $\left|\lambda_{\min }-\lambda_{\max }\right| \leq \epsilon$, stop. Otherwise, goto Step 2).

Note that a desired accuracy of $\epsilon$ in $\lambda$ translates directly to a desired accuracy in $\left(S_{1}, \ldots, S_{K}\right)$ and consequently a desired accuracy in sum capacity. This is because $g(\lambda)$, as a convex function defined on a closed and bounded interval $\left[\lambda_{\min }, \lambda_{\max }\right]$, has a bounded subgradient.

The proposed algorithm can also be viewed as an iterative method to solve the KKT condition of the original optimization problem. The KKT condition of the sum-power multiple-access channel sum capacity problem (8) consists of the stationarity condition

$$
\frac{1}{2} H_{i}^{T}\left(\sum_{k=1}^{K} H_{k} S_{k} H_{k}^{T}+S_{z}\right)^{-1} H_{i}=\lambda I+\Phi_{i}
$$

for $i=1, \ldots, K, \lambda \geq 0, \Phi_{i} \geq 0$, and the power constraint

$$
\sum_{k=1}^{K} \operatorname{tr}\left(S_{k}\right) \leq \mathrm{P}, \quad S_{k} \geq 0 .
$$

The dual decomposition method starts with a fixed $\lambda$, solves the set of (26), then adjusts $\lambda$ according to the search direction suggested by the power constraint. 


\section{B. Practical Implementation}

The proposed algorithm consists of two nested loops. In a practical implementation, it is not necessary to wait for the inner loop to converge fully in each iteration of the outer loop. This section contains an analysis of the accuracy with which the inner loop must reach and a modification of the above algorithm for practical implementation.

Instead of working with the dual variable $\lambda$ directly, it is easier to recast the problem in terms of a new variable $l=\frac{1}{2 \lambda}$, which has the interpretation of being the "water level" in the water-filling process. A bisection search on $l$ is numerically easier to analyze than a bisection search on $\lambda$. The strategy is not to run the inner iterative water-filling loop to full convergence, but only to a tolerance $\epsilon^{\prime}$ in total power, which depends on the length of $\left[l_{\min }, l_{\max }\right]$. In each bisection step, instead of reducing the length of feasible $\left[l_{\min }, l_{\max }\right]$ by a factor of 0.5 , a practical implementation of the algorithm reduces the length by a factor of $(0.5-$ $\nu)$, where $0<\nu<0.5$. At a new water level of $l=\left(l_{\min }+l_{\max }\right) / 2$ in one step of the bisection, the iterative water-filling process only has to return a sum power that is as accurate as an equivalent accuracy of $\pm \nu\left(l_{\max }-l_{\min }\right)$ in $l$. In practice, a value of $\nu=0.1$ works well.

Consider the sum power $p_{\text {total }}=\sum_{k=1}^{K} p_{k}^{*}$ as a function of $l$ in the inner iterative water-filling loop. Since $p_{\text {total }}$ is a continuous and strictly increasing function of $l$ defined on a closed and bounded interval $\left[l_{\min }, l_{\max }\right]$, its subgradient is bounded below. Let $\frac{\partial p_{\text {total }}}{\partial l}$ denote the subgradient; let $d$ be a lower bound on the subgradient, i.e., $\frac{\partial p_{\text {total }}}{\partial l} \geq d$. Then, to guarantee a level of accuracy of $\pm \nu\left(l_{\max }-l_{\min }\right)$ in $l$ in the inner loop, an equivalent accuracy in $p_{\text {total }}$ can be set as $\epsilon^{\prime}=\nu\left(l_{\max }-l_{\min }\right) d$. Thus, if the inner iterative water-filling loop uses the sum power as the terminating criterion with a tolerance set to $\epsilon^{\prime}$, then the outer loop is guaranteed to converge. Such a criterion is easy to achieve in the iterative water-filling inner loop, because coordinate descent is known to have an exponential convergence rate [10].

It remains to bound $d$. The value of $d$ is problem specific. However, in many situations, $\frac{\partial p_{\text {total }}}{\partial l}$ can be easily evaluated. Consider, for example, a broadcast channel with a large number of users and with a high signal-to-noise ratio (SNR). Intuitively, the optimal transmission scheme involves a subset of users with good channels and with low interference among them. In this case, let $r_{k}$ be the rank of the equivalent channel for user $k$ with a channel matrix $H_{k}$ and a noise covariance matrix $S_{z}+\sum_{j \neq k} H_{j} S_{j} H_{j}^{T}$. By the high SNR assumption, all $r_{k}$ subchannels are active. Since $p_{k}^{*}$ is the total power resulting from a water-filling, it is easy to see that $\frac{\partial p_{k}^{*}}{\partial l} \approx r_{k}$. This is because on each subchannel, if the channel noise is fixed, then the power allocation is a linear function of the water-filling level with a slope 1 . In a matrix channel with $r_{k}$ subchannels, the following holds: $\frac{\partial p_{k}^{*}}{\partial l} \approx r_{k}$. Therefore, $\frac{\partial p_{\text {total }}}{\partial l} \approx \sum_{k} r_{k}$, and $d$ can be set to be a fraction of $\sum_{k} r_{k}$. When fewer than $r_{k}$ subchannels are allocated positive power, the expression can be suitably modified.

In practice, it is easy to numerically evaluate $p_{\text {total }}$ over the range of $\left[l_{\text {min }}, l_{\text {max }}\right]$ to obtain a bound on $\frac{\partial p_{\text {total }}}{\partial l}$. For the simulation in the next section, it was found that $d=\frac{1}{4} \operatorname{rank}(H)$ works very well.

The modified algorithm is summarized below:

Algorithm 2: Computation of the sum-power constrained Gaussian multiple-access channel sum capacity.

1) Initialize $l_{\min }$ and $l_{\max }$.

2) Set $l=\left(l_{\min }+l_{\max }\right) / 2$.

3) Set $\epsilon^{\prime}=\left(l_{\max }-l_{\min }\right) \cdot \nu \cdot \operatorname{rank}(H) / 4$

4) Solve for $\left(S_{k}^{*}, p_{k}^{*}\right)_{k=1}^{K}$ in the optimization problem (14) with $\lambda=\frac{1}{2 l}$ by iteratively optimizing each of $\left(S_{k}, p_{k}\right)$ using (19)-(23) while keeping all other $\left(S_{k}, p_{k}\right)$ 's fixed. The iterations cycle through $k=1, \ldots, K, 1, \ldots, K, \ldots$ until the sum power is within $\epsilon^{\prime}$ of the optimal solution.
5) If $\sum_{k=1}^{K} p_{k}^{*}>\mathrm{P}$, then set

$$
l_{\max }=l_{\max }-\left(l_{\max }-l_{\min }\right) \cdot(0.5-\nu):
$$

else set

$$
l_{\min }=l_{\min }+\left(l_{\max }-l_{\min }\right) \cdot(0.5-\nu) .
$$

6) If $\left|\lambda_{\min }-\lambda_{\max }\right| \leq \epsilon$, stop. Otherwise, goto Step 2).

\section{NUMERICAL SIMULATION}

The iterative-water-filling-based algorithm proposed in this correspondence exploits the structure of the optimization problem and is much faster than generic gradient-search-based algorithms [9] or interior-point methods. In this section, the focus is on the comparison of the proposed dual decomposition algorithm with the other two iterative-water-filling-based algorithms proposed in [7].

The algorithms in [7] are motivated as follows. Instead of updating the noise covariance matrix as soon as a water-filling step for a single user is taken, the algorithms in [7] perform a single water-filling over all $K$ channels with a single power constraint $P$. This water-filling step ensures that the total power is always $P$, thus eliminating the need for an outer power update. However, in order to ensure convergence, the update of the covariance matrices must be taken as an average of the newly obtained water-filling covariance with the past $K-1$ covariance matrices. This update rule is named "Algorithm One" in [7]. "Algorithm One" has an $O\left(K^{2}\right)$ memory requirement because $O(K)$ covariance matrices need to be stored in the memory for each of the $K$ users. To obtain an algorithm with $O(K)$ memory requirement, [7] further proposes a different update rule in which the new water-filling covariance is averaged with the immediate past covariance matrix only. However, the newly obtained covariance is given a weight of $\frac{1}{K}$, and the immediate past covariance is given a weight of $\frac{K-1}{K}$. This second update rule is named "Algorithm Two." In the following discussion, the two algorithms proposed in [7] are copmpared with the algorithm proposed in Section II-B of this correspondence, which is referred to as the "dual decomposition" algorithm.

Fig. 1 illustrates the convergence behavior of the proposed dual decomposition algorithm as compared with "Algorithm One" and "Algorithm Two" of [7]. A broadcast channel in which the transmitter has 10 antennas and each receiver has 2 antennas is considered. Each entry of the channel matrix is an i.i.d. Gaussian random variable with mean 0 and variance 1 . The total power constraint is set to be 10 . Two scenarios with the number of users $K=10$ and $K=50$ are simulated. Each iteration of the three algorithms consists of an equivalent of $K$ individual water-filling steps. The horizontal segments in the curve for the dual decomposition algorithm represent the number of iterations for each fixed $\lambda$. The staircase shape of the curves is indicative of the bisection algorithm on $\lambda$.

First, it was observed that all three algorithms converge to the correct sum rate. However, while the algorithms proposed in [7] approach the correct sum rate faster at the beginning, they suffer from a poorer asymptotic convergence behavior for systems with a large number of users. With $K=10$, the proposed dual decomposition algorithm converges at about the same rate as "Algorithm One", but converges faster than "Algorithm Two". (The comparison with "Algorithm One" is problem specific. Both cases in which the dual decomposition algorithm outperforms "Algorithm One" and cases in which "Algorithm One" outperforms the dual decomposition algorithm are observed.) As both the dual decomposition algorithm and "Algorithm Two" require only $O(K)$ memory while "Algorithm One" requires $O\left(K^{2}\right)$ memory, the comparison with "Algorithm Two" is more fair. With $K=50$, the dual decomposition algorithm outperforms both "Algorithm One" and 

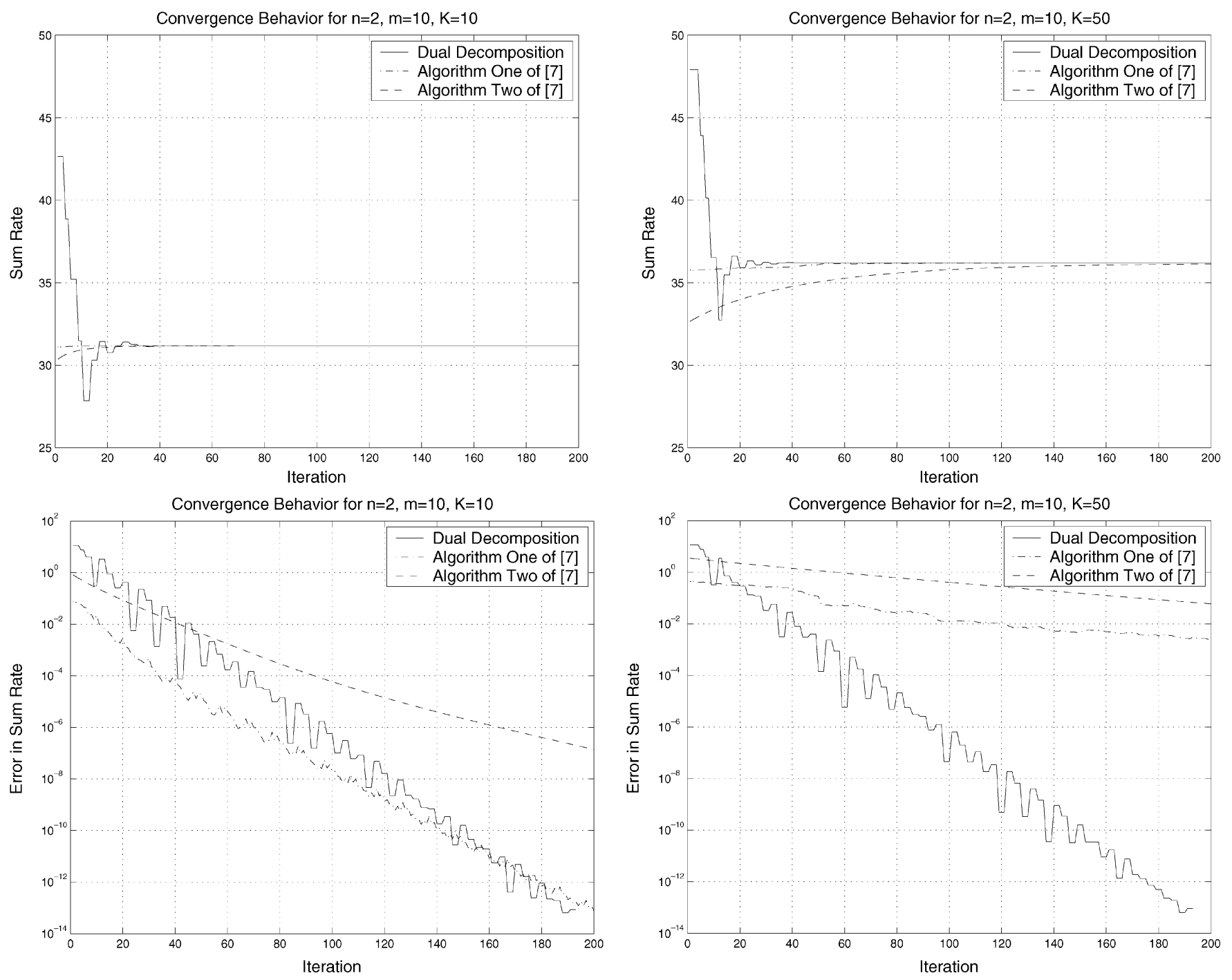

Fig. 1. Comparison of numerical algorithms for sum capacity computation of the Gaussian vector broadcast channel. The transmitter has $m=10$ antennas. Each receiver has $n=2$ antennas. The convergence behavior of various algorithms for a system with $K=10$ users is plotted on the left. The convergence behavior for a system with $K=50$ users is plotted on the right.

"Algorithm Two" significantly. The apparent initial faster convergence of "Algorithm One" and "Algorithm Two" is due to the fact that both algorithms always satisfy the power constraint exactly in each iteration, while the dual decomposition algorithm requires several iterations to zoom in on a correct power level.

The proposed dual decomposition algorithm also has the very desirable property that its asymptotic convergence rate appears to be independent of $K$. As the complexity per iteration scales linearly with $K$, the computational complexity of the algorithm is roughly $O(K)$, which is important for a system with a large number of users. This convergence behavior is due to the fact that the proposed algorithm solves the problem in the dual domain in which $g(\lambda)$ is always one dimensional regardless of the number of users in the system. The asymptotic convergence rates of algorithms proposed in [7] are highly problem-size dependent. The total computational complexity of the algorithms proposed in [7] does not scale linearly with $K$.

The advantage of the proposed dual decomposition algorithm over the algorithms proposed in [7] for systems with a large number of users is significant, because this is also the regime in which the benefit of transmit optimization is the largest. In a downlink system, in which the number of users is much larger than the number of transmit antennas, the transmit optimization process implicitly selects the best subset of active users. The improvement in system performance due to the selection of an optimal set of active users is commonly referred to as the benefit of multiuser diversity. The dual decomposition algorithm proposed in this correspondence gives an efficient way to evaluate the benefit of multiuser diversity in term of sum capacity.

Finally, it should be noted that each iteration in the proposed dualdecomposition algorithm consists of $K$ individual water-filling steps, which can be performed in $O(K)$ operations. In contrast, each iteration in the two algorithms proposed in [7] requires a simultaneous water-filling over $O(K)$ subchannels, which requires $O(K \log (K))$ operations. Thus, the asymptotic complexity per iteration for the dual decomposition algorithm is also lower than that of the algorithms in [7].

\section{CONCLUSION}

This correspondence proposes a numerical solution to solve for the sum capacity of a Gaussian vector broadcast channel. The broadcast channel is first transformed into a sum-power constrained Gaussian vector multiple-access channel, for which an iterative-water-filling based algorithm is proposed to compute its sum capacity. The main feature of the proposed algorithm is a dual decomposition approach 
that decouples the sum-power constraint. The proposed algorithm exhibits a faster asymptotic convergence rate and a lower computational complexity than comparable algorithms in the literature.

\section{REFERENCES}

[1] G. Caire and S. Shamai (Shitz), "On the achievable throughput of a multi-antenna Gaussian broadcast channel," IEEE Trans. Inf. Theory, vol. 49, no. 7, pp. 1691-1706, Jul. 2003.

[2] W. Yu and J. M. Cioffi, "Sum capacity of Gaussian vector broadcast channels," IEEE Trans. Inf. Theory, vol. 50, no. 9, pp. 1875-1892, Sep. 2004.

[3] P. Viswanath and D. Tse, "Sum capacity of the multiple antenna Gaussian broadcast channel and uplink-downlink duality," IEEE Trans. Inf. Theory, vol. 49, no. 8, pp. 1912-1921, Jul. 2003.

[4] S. Vishwanath, N. Jindal, and A. Goldsmith, "Duality, achievable rates and sum-rate capacity of Gaussian MIMO broadcast channels," IEEE Trans. Inf. Theory, vol. 49, no. 10, pp. 2658-2668, Oct. 2003.

[5] H. Weingarten, Y. Steinberg, and S. Shamai (Shitz), "The capacity region of the Gaussian MIMO broadcast channels," IEEE Trans. Inf. Theory, to be published.

[6] W. Yu, W. Rhee, S. Boyd, and J. M. Cioffi, "Iterative water-filling for Gaussian vector multiple access channels," IEEE Trans. Inf. Theory, vol. 50, no. 1, pp. 145-151, Jan. 2004.

[7] N. Jindal, W. Rhee, S. Vishwanath, S. A. Jafar, and A. Goldsmith, "Sum power iterative water-filling for multi-antenna Gaussian broadcast channels," IEEE Trans. Inf. Theory, vol. 51, no. 4, pp. 1570-1580, Apr. 2005.

[8] L. Xiao, M. Johansson, and S. P. Boyd, "Simultaneous routing and resource allocation via dual decomposition," IEEE Trans. Commun., vol. 52, no. 7, pp. 1136-1144, Jul. 2004.

[9] H. Viswanathan, S. Venkatesan, and H. Huang, "Downlink capacity evaluation of cellular networks with known-interference cancellation," IEEE J. Sel. Areas Commun., vol. 21, no. 5, pp. 802-811, Jun. 2003.

[10] Z. Q. Luo and P. Tseng, "On the convergence of the coordinate descent method for convex differentiable minimization," J. Opt. Theory Applic., vol. 72, no. 1, pp. 7-35, Jan. 1992.

\section{An Asymptotic Analysis of Band-Limited DS/SSMA Communication Systems}

Yeon K. Jeong, Member, IEEE, Joon Ho Cho, Member, IEEE, and James S. Lehnert, Fellow, IEEE

Abstract-A new asymptotic analysis is presented to establish whether or not commonly used techniques to approximate the performance of direct-sequence spread-spectrum multiple-access (DS/SSMA) systems still apply when the systems are strictly band limited. The results apply to any linear data modulation and spreading scheme. The interference components, including the interchip interference (ICI) of a linear receiver output, are shown to be asymptotically jointly Gaussian random variables conditioned on the phases and delays of the interfering signals. Expressions for the error probabilities of band-limited systems are also developed from this result.

Index Terms-Approximation theory, asymptotic analysis, code-division multiple access, interference.

\section{INTRODUCTION}

Analysis of asynchronous direct-sequence spread-spectrum multiple-access (DS/SSMA) communication systems has long been a topic of interest. The objectives of the analysis have been to identify key design parameters and to understand how the parameters affect the system performance. The difficulty in the analysis is that finding the exact probability distribution of the decision variable is not feasible in many cases, and that, even if this is possible, the exact distribution may not identify the key design parameters and may not reveal how the parameters affect the system performance. Hence, research on the analysis of asynchronous DS/SSMA systems has been focused on deriving simple and accurate approximation methods that satisfactorily accomplish the objectives of the performance analysis. Gaussian approximations to the overall interference at the output of linear receivers are commonly used to analyze the performance of DS/SSMA communication systems. However, since the validity of the approximation methods has been established mainly for the systems employing time-limited chip waveforms, they do not necessarily apply to band-limited systems where the durations of chip waveforms are much longer than the reciprocal of the chip rate. In this work, a new asymptotic analysis is presented to establish the validity of Gaussian approximations for the systems employing more general chip waveforms of interest, which include strictly band-limited waveforms.

The simplest among the Gaussian approximations developed so far is the standard Gaussian approximation (SGA) [1], where the overall interference in the decision variable is approximated as a Gaussian noise. The SGA is justified for asynchronous DS/SSMA [5] and for symbol-

Manuscript received April 15, 2004; revised May 10, 2005. The material in this correspondence was presented in part at the IEEE International Symposium on Information Theory, Chicago, IL, June/July 2004.

Y. K. Jeong was with the School of Electrical and Computer Engineering, Purdue University, West Lafayette, IN 47907 USA. He is now with Qualcomm Inc., Boulder, CO 80301 USA (e-mail: yjeong@qualcomm.com).

J. H. Cho was with the Department of Electrical and Computer Engineering, University of Massachusetts, Amherst, MA 01003 USA. He is now with the Department of Electronic and Electrical Engineering, Pohang University of Science and Technology (POSTECH), Pohang, Kyungbuk 790-784, Korea (e-mail: jcho@postech.ac.kr).

J. S. Lehnert is with the School of Electrical and Computer Engineering, Purdue University, West Lafayette, IN 47907 USA (e-mail: lehnert@purdue. edu).

Communicated by R. R. Müller, Associate Editor for Communications.

Digital Object Identifier 10.1109/TIT.2005.860470 